\title{
QUALITY CONTROL OF GRAPHENE OXIDE PRODUCTION WITH LARGE AREA SHEETS
}

\author{
1,2Pavel SVEC, 1,2Zuzana BYTESNIKOVA, 1,2Vojtech PRIKRYL, 2Jana PEKARKOVA, \\ ${ }^{1}$ Lenka VANKOVA, ${ }^{2}$ Sanam GAREHBAGHI, 1,2Lukas RICHTERA, 1,2Vojtech ADAM \\ ${ }^{1}$ Department of Chemistry and Biochemistry, Mendel University in Brno, Brno, Czech Republic, EU, \\ svecpavel@centrum.cz \\ ${ }^{2}$ Central European Institute of Technology, Brno University of Technology, Brno, Czech Republic, EU, \\ svecpavel@centrum.cz
}

https://doi.org/10.37904/nanocon.2021.4319

\begin{abstract}
There are many processes and modifications for the production of graphene oxide (GO) that are based on the original synthesis by W.S.Hummers and R.E.Offeman (1957), B.C.Brodie (1859), L.Staudenmaier (1898). Not all modifications produce high-quality material. The GO production process we use is a modified production according to Mr. W.S.Hummers and Mr. R.E.Offeman. Because the synthesis itself consists of several individual steps each step must be controlled in order to achieve the best result. Quality control at every step helps us to reduce the content of impurities and increase the yield of the final product. Impurities can be toxic or electrochemically active and thus have a direct effect on the application of the produced material. The ideal result is that the final product will contain only GO with individual large sheets and will not contain any other impurities and byproducts from synthesis such as potassium, chlorine, sulfur, manganese salts, or nonexfoliated graphite oxide. At the same time, we must reach sufficient production and small losses. We use Scanning Electron Microscopy with Energy Dispersive X-ray Analysis (SEM-EDX), conductometer, and pH meter to control the individual steps. The result of our production is single layer $\mathrm{GO}$ with large area sheets.
\end{abstract}

Keywords: Graphene oxide, large area sheets, impurities, nanomaterials, synthesis

\section{INTRODUCTION}

Graphene oxide (GO) was first synthesized by Brodie in 1855 [1]. Later, Hummers and Offeman [2] improved its production. GO is a promising material applied in many industries, such as the food, electrical, health, and agricultural sectors. The GO is involved in packaging material due to its modifying agents in the food industry. It can increase the surface of the electrodes, while in electrochemistry devices such as biosensors and a protective layer while developing anodes in the electrical industry. It can form composites with other nanomaterials having adhesive and antibacterial properties, suitable for healthcare instruments. The antibacterial and antifungal properties of GO have encouraged to be applied in the agricultural sector also $[3,4,5]$.

The reasons have encouraged us to start our production of GO due to low quality and increasing prices of commercially available GOs from different manufacturers. These commercial GOs contained many impurities such as fibers, residues of nano-oxidized graphite, nano-exfoliated graphite oxide, unwashed salts, and other undefined contaminants. Despite the proclaimed sizes of individual GO sheets, the sheets were not homogenous, having significant differences in size and quality. 


\section{MATERIALS AND METHODS}

\subsection{Chemicals}

Chemicals used in this study (graphite flakes, $\mathrm{H}_{2} \mathrm{SO}_{4}, \mathrm{HCl}, \mathrm{KMnO}_{4}, \mathrm{H}_{2} \mathrm{O}_{2}$ ) were purchased from Sigma-Aldrich (St. Louis, MO, USA) in ACS purity. Deionized water was purified using a Milli-Q Direct QUV apparatus equipped with a UV lamp (Merck Millipore, Darmstadt, Germany). The resistance was $18.2 \mathrm{M} \Omega$ [5].

\section{2. $\mathrm{pH}$ and conductivity}

The pH meter inoLab pH 720 (WTW, Wellheim, Germany) was used for the $\mathrm{pH}$ measurement with a standard pH electrode. A conductometer inoLab Cond7110 (Xylem Analytics, Wellheim, Germany) was used to measure conductivity with a common electrode where the cell volume was $1 \mathrm{~cm}^{3}$ and temperature was $21^{\circ} \mathrm{C}$. The conductivity and $\mathrm{pH}$ measurements were done while washing the $\mathrm{GO}$ with $\mathrm{HCl}$. A shallow $\mathrm{pH}$ value was observed during synthesis.

\subsection{Scanning electron microscopy}

The well-dispersed GO sample solution was prepared by dilution. The dilution ratio was 1:200 with demineralized water, and then the sample was drop cast on a silicon wafer and allowed to dry at laboratory temperature $\left(22^{\circ} \mathrm{C}\right)$. This wafer was adhered by carbon tape to the stub and later inserted into the SEM. The morphology and composition of the GO were examined by scanning emission microscopy with a Tescan MAIA 3 equipped with a field emission gun (Tescan Ltd., Brno, Czech Republic). The best images were obtained using the In-Lens SE detector at a working distance between $3-5 \mathrm{~mm}$ and $5 \mathrm{kV}$ acceleration voltage. A pixel image of $768 \times 858$ dimensions was obtained at $5,000-50,000$ fold magnification. In UH Resolution mode, a full-frame was captured, and the accumulation of image with image shift correction was enabled, and it took about 1.5 minutes with the $\sim 1 \mu \mathrm{s} /$ pixel dwell time. The $2.4 \mathrm{~nm}$ spot size was set.

\subsection{Synthesis of GO}

The GO was synthesized by following the procedure reported earlier in Richtera et al. 2015 [5]. Each synthesizing steps of GO were monitored by SEM, including the graphite that entered into the reaction.

\subsubsection{Start of production (green phase)}

The graphite flakes were mixed with $\mathrm{H}_{2} \mathrm{SO}_{4}$, and then $\mathrm{KMnO}_{4}$ was added. Graphite was placed in water for SEM image only to ensure the same condition (liquid medium). Graphite entered the production process in dry form. The resulting reaction mixture was green. At each addition, a sample was taken and characterized in SEM (Figure 1).

\subsubsection{Oxidation at time and termination (brown and pink phase)}

The green reaction mixture was stirred for four days until the green color changed to brown. After four days, the mixture was allowed to stand for another five days until it turned into pink color. $\mathrm{H}_{2} \mathrm{O}_{2}$ was used to terminate the reactions (Figure 2).

\subsubsection{Washing with $\mathrm{HCl}$ and water}

A color change manifested the completed reactions from pink to yellow. This was followed by a gradual and intensive washing with $\mathrm{HCl}$ (Figure 3). Eventually, water started to be added to separate the $\mathrm{GO}$ sheets because the water intercalated into the layers of graphite oxide (Figure 4). 


\section{RESULTS}

\subsection{SEM observation}

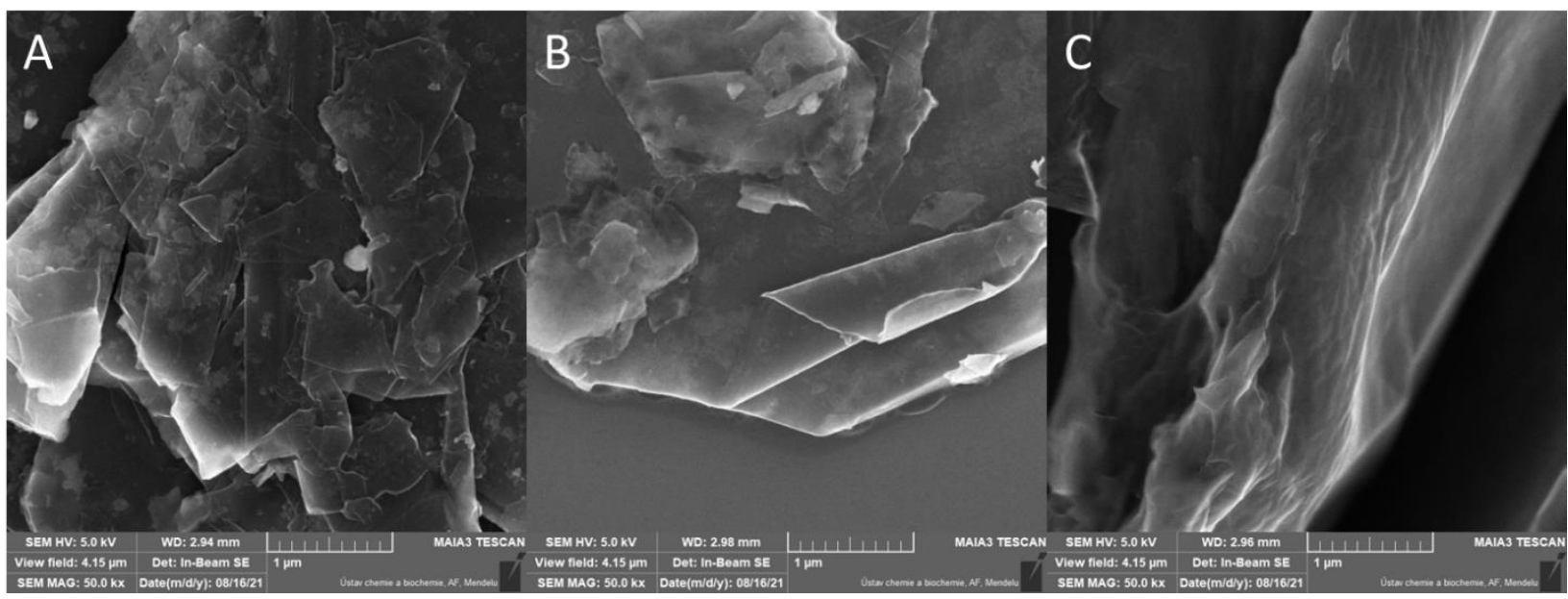

Figure 1 Graphite flakes in green phase. A - graphite flakes, B - graphite flakes with $\mathrm{H}_{2} \mathrm{SO}_{4}, \mathrm{C}$ - graphite flakes with $\mathrm{H}_{2} \mathrm{SO}_{4}$ and $\mathrm{KMnO}_{4}$.

In Figure 1A, we can see the structure of graphite entering the reaction. A large block of graphite flakes consists of smaller and thinner sheets stacked on top of each other. The surface is smooth and flat, without wrinkles or other deformations. Figure 1B, after the addition of $\mathrm{H}_{2} \mathrm{SO}_{4}$, shows the edge of one graphite flakes, where the sandwich structure is visible. By sandwich, the system is meant the composition of many $2 \mathrm{D}$ sheets of graphite. After the addition of $\mathrm{KMnO}_{4}$, the individual graphite layers oxidized (Figure 1C). It is most noticeable on the surface. The original smooth surface of graphite is wrinkled. This wrinkling is due to the local separation of the individual graphite layers from each other during oxidation. Oxidation proceeds most rapidly at the edges and outer layers and progresses to the depth of the flakes [6].

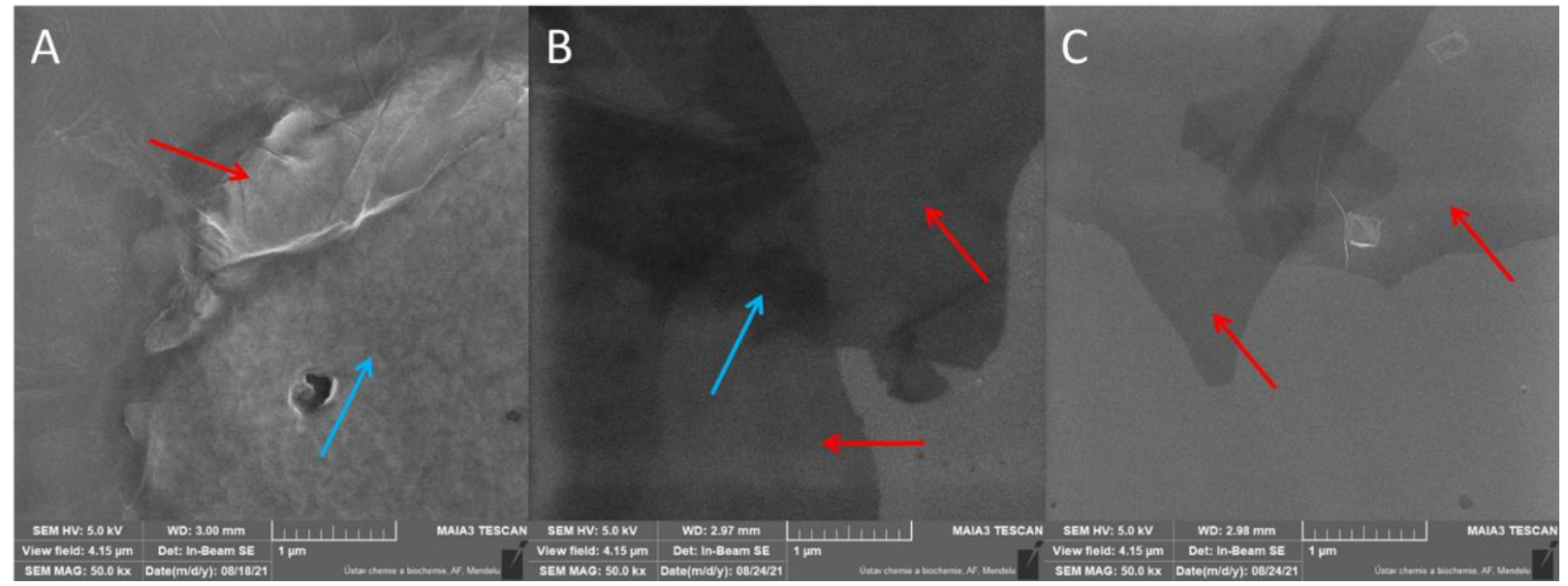

Figure 2 Oxidation phase of graphite. A - graphite flakes after four days of oxidation, B - graphite oxide sheets before termination, $\mathrm{C}$ - graphite oxide after termination.

Four days of oxidation of graphite with constant stirring helped break down the sandwich structure into thinner layers. In Figure 2A (red arrow), we see a smaller piece of graphite. This piece has no sharp edges and is wrinkled. The individual thinner layers of graphite oxide slip from the original graphite on the upper and left sides. The blue arrow indicates where the graphite oxide layer covered the salts formed in the reaction. This 
layer of graphite oxide prevented the formation of larger salt crystals. The reaction mixture stood for the next five days. During this time, the graphite flakes oxidized, and the block of sheets was separated. Figure 2B shows the block of sheets (red arrows) with a large area and not torn. The blue arrow indicates the residues of the reaction mixture between the two graphite oxide sheets. In Figure $\mathbf{2 C}$ we see graphite oxide after termination. Upon termination with hydrogen peroxide, the oxidation number changed from $\mathrm{Mn}^{+\mathrm{VII}}$ to $\mathrm{Mn}^{+11}$. This reaction altered the color of the suspension from a pink to a yellow, clear liquid containing thin layers of graphite oxide [6].

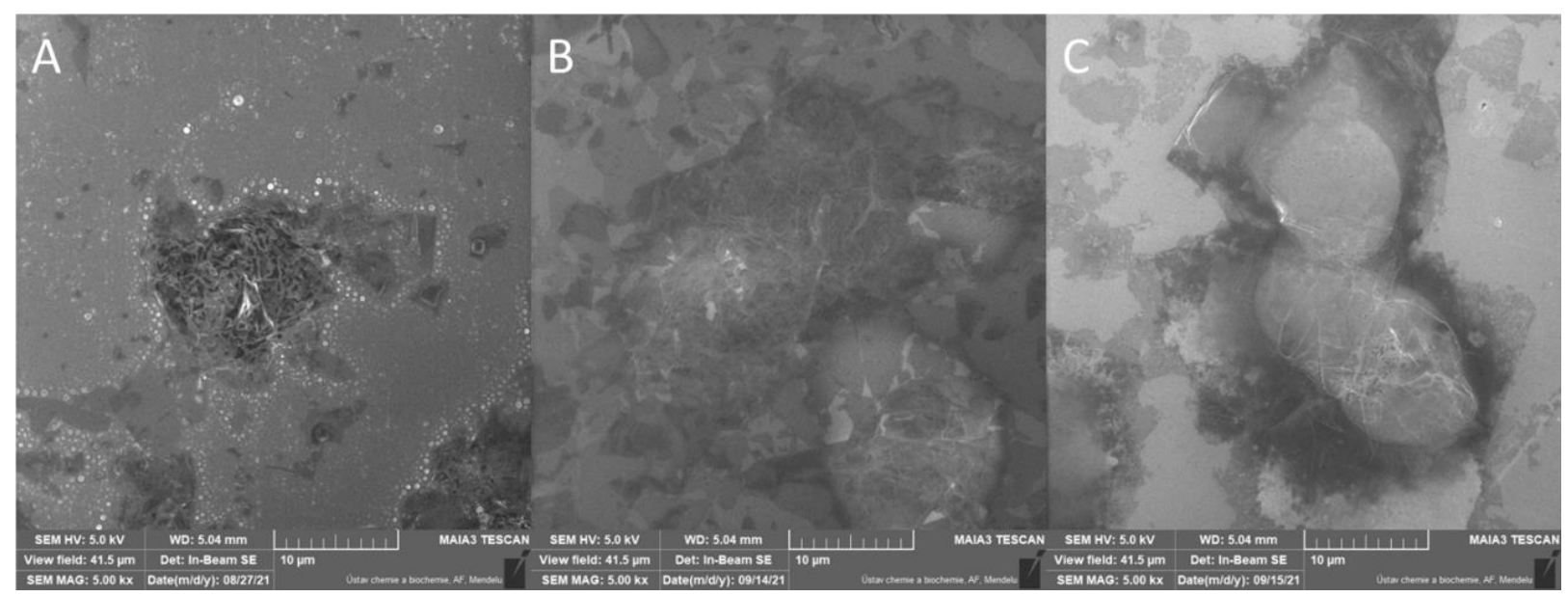

Figure 3 Washing graphite oxide with $\mathrm{HCl}$. A - graphite oxide with impurities, $\mathrm{B}$ - the mount of impurities decreases, $\mathrm{C}-$ state of graphite oxide at the end of washing with $\mathrm{HCl}$.

As we can see in Figure 3A, the sample contains many salts in the form of crystals. These are $\mathrm{MnCl}_{2}$ and $\mathrm{MnSO}_{4}$. Gradual washing with $\mathrm{HCl}$ reduces the amount of $\mathrm{Mn}^{+11}$ bound to the graphite oxide. The gradual decrease of $\mathrm{Mn}^{+11}$ can be seen in Figure 3B, where almost no crystals of the formed salts are visible. However, we can see areas where acid residues under the graphite oxide layer are. These residues are well visible in Figure 3C, where they have an ellipse shape and are covered with graphite oxide [6].

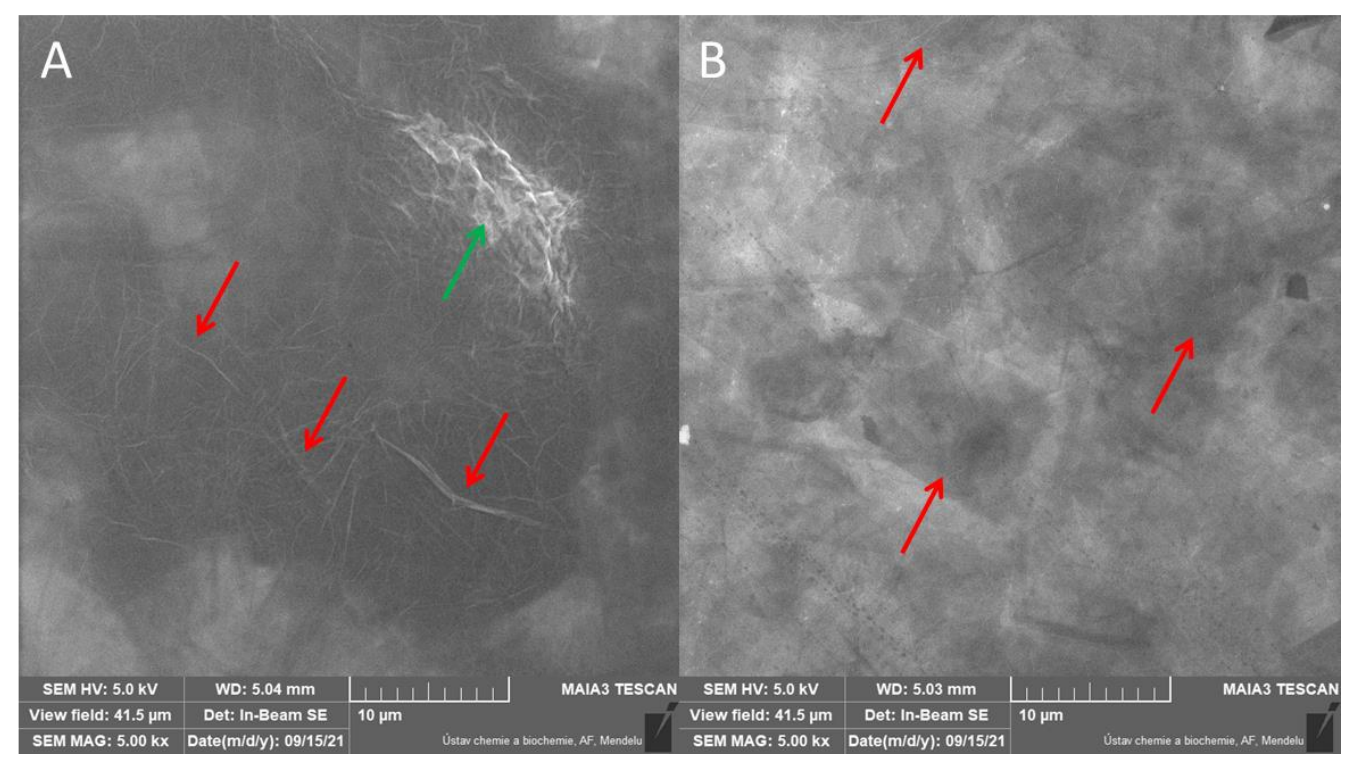

Figure 4 Separating individual GO sheets. A - after $8 \mathrm{~L}$ of water, B - after $16 \mathrm{~L}$ of water. The red arrows show the wrinkles that occur when the individual leaves of the $\mathrm{GO}$ are separating. The green arrow indicates where the acid residues are. 
At the beginning of the washing with water (first wash), acid residues were still present in the sample, as seen in Figure 4A (green arrow). The red arrows show where the wrinkles of GO are visible. There are also many layers of GO, which lie loosely on top of each other and no longer hold in the block as previously with graphite flakes. After further washing with water, we no longer see any acid residues, and the exfoliation of the individual GO layers continues, as shown in Figure 4B. Red arrows indicate a fine wrinkle of the GO [6].

\section{2. $\mathrm{pH}$ and conductivity}

Following individual washing phases, $\mathrm{pH}$ and conductivity values of $\mathrm{GO}$ were measured (Table 1). The $\mathrm{HCl}$ fractions were diluted 10 -fold before measuring the $\mathrm{pH}$ because these fractions were too acidic.

Table 1 Values of $\mathrm{pH}$ and conductivity

\begin{tabular}{|c|c|c|}
\hline Sample & $\mathbf{p H}$ & Conductivity (mS/cm) \\
\hline 1. fraction of $\mathrm{HCl}$ & 0.52 & 379.00 \\
\hline 2. fraction of $\mathrm{HCl}$ & 0.64 & 360.00 \\
\hline 3. fraction of $\mathrm{HCl}$ & 0.75 & 320.00 \\
\hline 1. fraction of water & 0.52 & 83.60 \\
\hline 2. fraction of water & 1.41 & 13.13 \\
\hline
\end{tabular}

Increasing $\mathrm{pH}$ values and decreasing conductivity values show that the number of ions released in previous synthesis steps (impurities) decreases, indicating a successful cleaning. These changes were visible in the Figures 3A, B, C, and 4A, B. In $\mathrm{HCl}$ fractions $(1-3)$, the $\mathrm{pH}$ increase very slowly. In these 3 fractions, the conductivity slowly decreases because only $\mathrm{Mn}$ ions are removed, but the anions from acids remain in excess. In the individual washing steps with water, we noticed that the conductivity continued to decrease sharply due to continuously removing anions through washing following a constant increase in the $\mathrm{pH}$.

\section{CONCLUSION}

The modified Hummers method is very effective in the production of GO. The individual synthesis steps and gradual improvement allowed us to produce GO sheets with a large surface. The synthesized GO has low impurities after washing. The decreasing conductivity and simultaneous increasing $\mathrm{pH}$ during each washing step confirm the removal of impurities. SEM monitored each step of GO synthesis and cleaning. Corresponding changes in SEM images of GO were observed during individual cleaning steps and the following measurement in conductometer and $\mathrm{pH}$ value. The quality of the synthesis was continuously monitored from graphite to GO along with the course of oxidation. Continual quality check with SEM helped clarify the oxidation of the outer layers of graphite, the separation of individual blocks of graphite oxide, and the effect of washing after the oxidation to the GO.

\section{ACKNOWLEDGEMENTS}

This research was carried out under the project CEITEC 2020 (LQ1601) with financial support from the Ministry of Education, Youth and Sports of the Czech Republic under the National Sustainability Programme II.

The work was supported from ERDF " Multidisciplinary research to increase application potential of nanomaterials in agricultural practice " (No. CZ.02.1.01/0.0/0.0/16_025/0007314). 


\section{REFERENCES}

[1] BRODIE, B.C., XII. On the atomic weight of graphite. Philosophical transactions of the royal society of London. 1859, vol. 149, pp. 249-259.

[2] HUMMERS, W.S., OFFEMAN, R.E. Preparation of graphitic oxide. Journal of the American Chemical Society. 1958, vol. 80, pp. 1339.

[3] ZHU, Y., JAMES, D.K., TOUR, J.M. New routes to graphene, graphene oxide and their potential applications. Advanced Materials. 2012, vol. 24, pp. 4924-4955.

[4] KIM, J., COTE, L.J., HUANG, J., Two dimensional soft materials: New faces of graphene oxide. Accounts of chemical research. 2012, vol. 45, pp. 1356-1364.

[5] RICHTERA, L., et. al. The composites of graphene oxide with metal or semimetal nanoparticles and their effect on pathogenic microorganisms. Materials. 2015, vol. 8, no. 6, pp. 2994-3011.

[6] DIMIEV, A.M., TOUR, J.M. Mechanism of graphene oxide formation. ACSNANO. 2014, vol. 8, pp. 3060-3068. 